Meta

Journal des tradlucteurs

Translators' Journal

\title{
La recherche thématique à Hydro-Québec
}

\section{Francine Doray et Rollande Gaudet}

Volume 36, numéro 1, mars 1991

La terminologie dans le monde : orientations et recherches

URI : https://id.erudit.org/iderudit/002579ar

Aller au sommaire du numéro

Éditeur(s)

Les Presses de l'Université de Montréal

\section{ISSN}

0026-0452 (imprimé)

Découvrir la revue

Citer cet article

Doray, F. \& Gaudet, R. (1991). La recherche thématique à Hydro-Québec. Meta, 36(1), 243-247. d'utilisation que vous pouvez consulter en ligne.

https://apropos.erudit.org/fr/usagers/politique-dutilisation/ 


\title{
LA RECHERCHE THÉMATIQUE À HYDRO-QUEBEC
}

\author{
Francine Doray et Rollande Gaudet \\ Hydro-Québec, Montréal, Canada
}

À Hydro-Québec, plus particulièrement au service Terminologie et Diffusion, la terminologie comprend plusieurs volets : consultations ponctuelles, expertise et validation des documents officiels de l'entreprise, promotion de la qualité de la langue par le truchement des chroniques Pour bien se comprendre publiées dans le journal de l'entreprise. S'y greffent la tâche de veiller à l'application des directives de la Charte de la langue française, ainsi que des règles et des normes de la Commission de toponymie du Québec.

L'activité que nous privilégions cependant est la recherche thématique. Une entreprise dont la langue de travail est le français se doit en effet d'uniformiser et de normaliser sa terminologie technique, scientifique et administrative. Et il revient au service de fournir une terminologie de qualité, gage de la cohérence et de l'efficacité des communications tant internes qu'externes.

Nous réalisons des glossaires, des mini-thématiques, et nous rédigeons des études terminologiques. Toutefois, pour normaliser et uniformiser la terminologie, ce sont les lexiques et les vocabulaires, plus particulièrement les vocabulaires unilingues, qui répondent le mieux aux besoins de l'entreprise.

\section{POURQUOI DES THÉMATIQUES?}

Le besoin de créer des vocabulaires et des lexiques s'est fait sentir dès le début des années 70. Mais c'est à la fin de cette décennie que nous avons entrepris de systématiser la mise au point de ces documents. Les nombreuses demandes de révision de documents techniques portant sur les lignes aériennes ont mis en évidence la grande disparité des termes employés par les ingénieurs et les spécialistes. Ce qui nous a amenés à élaborer un vocabulaire illustré des lignes aériennes de transport et de distribution d'électricité. À cette fin, nous avons mis sur pied un comité de travail.

L'idée a fait boule de neige, suscitant l'intérêt des spécialistes des lignes souterraines qui, à leur tour, voulaient «leur vocabulaire». À la fin des années 80 donc, paraissait le Vocabulaire illustré des lignes souterraines de transport et de distribution d'électricité. Entre-temps le concept des vocabulaires avait touché d'autres domaines, la fonction commerciale, le contrôle de gestion et la turbine Francis, par exemple.

\section{ÉLABORATION D'UN VOCABULAIRE}

Nous traiterons ici d'un vocabulaire illustré en soulignant que la réalisation d'un lexique suit essentiellement les mêmes étapes. Élaborer un vocabulaire est un travail de longue haleine, qui peut s'étendre sur une ou deux années. Avant même de passer à la recherche terminologique proprement dite, il faut mettre sur pied un comité de travail, effectuer la recherche documentaire, constituer un arbre de domaine, établir et valider la nomenclature. Viennent ensuite les définitions et la préparation du manuscrit, suivies de la version provisoire, de la préparation du produit final qui débouchera sur l'édition et la diffusion. 


\section{FORMATION DU COMITÉ}

La première étape consiste à mettre sur pied le comité de référence. Il est formé d'une ou d'un terminologue, de deux parfois, et de spécialistes dont le nombre varie entre trois et sept selon les dossiers. Les spécialistes interviennent à maintes reprises au cours du travail terminologique, d'où l'importance attachée au choix des personnes. Les candidats idéals répondent à certains critères : excellente connaissance du domaine étudié, disponibilité, intérêt manifeste pour la langue et la qualité de la terminologie, et capacité de travailler en équipe. Nous avons constaté que la crédibilité et l'engagement des spécialistes contribuent à faire accepter le produit terminologique et favorisent son implantation dans le milieu de travail.

Nous insistons beaucoup sur le tandem terminologues et spécialistes, car nous estimons que l'uniformisation et la normalisation terminologiques reposent sur la concertation des principaux intéressés. Chaque spécialiste apporte son propre éclairage sur les termes et, par ses questions ou ses commentaires, aide à en préciser le contenu notionnel.

La coordination des réunions est généralement confiée à la personne responsable de la terminologie ou à une terminologue ou un terminologue d'expérience. Cette personne doit établir le calendrier de travail, assurer le suivi du dossier, faire le lien entre le service de terminologie et les unités concernées. En outre, de concert avec les responsables du graphisme, elle fixe l'échéancier de production et en fait le suivi. Finalement, elle établit la stratégie de diffusion du document avec les spécialistes en diffusion.

\section{DOCUMENTATION ET NOMENCLATURE}

Le travail de recherche s'amorce du côté de la documentation. Une recherche exhaustive est effectuée dans le domaine traité par les terminologues, avec l'assistance de la bibliothécaire du service. Le Réseau d'information documentaire automatisée d'HydroQuébec (RIDAQ) peut fournir une bibliographie des ouvrages du domaine. D'autres bases de données spécialisées sont également consultées. Des trésors non répertoriés dorment souvent dans les bureaux des spécialistes; ils serviront à enrichir le fonds documentaire.

La recherche documentaire donne souvent lieu à la construction d'un arbre de domaine. Comme le dit Robert Dubuc dans son Manuel pratique de terminologie, «l'arbre de domaine servira non seulement à structurer le vocabulaire, mais aussi à évaluer la pertinence des unités terminologiques et à restreindre les bruits (c'est-à-dire les informations parasites non liées au thème de la recherche) et les silences (c'est-à-dire les lacunes touchant l'information dont on aura besoin mais qui ne figure pas dans les termes de la recherche)». L'arbre de domaine pourra même servir à rédiger la table des matières du vocabulaire.

Les terminologues entreprennent ensuite le dépouillement de la documentation retenue afin d'établir la liste des termes à étudier. Il arrive aussi que leur nomenclature serve de complément à la liste présentée par les spécialistes. De toute façon, la nomenclature sera arrêtée au cours des premières réunions du comité. À mesure que le travail avance, certains termes peuvent être supprimés à la lumière des analyses en profondeur qui suivent. Le contraire se produit également lorsque les spécialistes ou les terminologues constatent des oublis.

\section{ÉLABORATION ET MISE EN FORME}

Puis, vient l'étape de la recherche terminologique même et de la consignation des données, point de départ des définitions. En plus des ouvrages dépouillés lors de la recherche documentaire, les terminologues consultent les grandes banques de données 
terminologiques ainsi que des collègues et des experts d'organismes et d'autres entreprises reconnus du domaine, comme Électricité de France.

$\hat{A}$ partir des données contextuelles et des définitions relevées, les terminologues ébauchent peu à peu des définitions. Leur formulation s'inscrit tout à la fois comme une synthèse et une adaptation. La définition doit respecter les critères de précision, de concision, de clarté et de simplicité. Il importe d'éviter les tournures savantes qui pourraient dérouter l'usager et le rebuter. Ce qui n'exclut pas pour autant la définition de termes très techniques destinés à des spécialistes.

Le service a convenu d'adopter la terminologie française en usage à l'échelle internationale, sauf lorsque des réalités spécifiquement nord-américaines amènent à s'en écarter et à proposer un terme nouveau ou un terme largement répandu. Ainsi en va-t-il par exemple du pylône Mae West appelé ailleurs pylône nappe ou pylône américain. En revanche, les terminologues doivent parfois redresser la terminologie lorsque le terme en usage est fautif. $\grave{A}$ titre d'exemple, le terme grimpette, dispositif que le monteur place sous chacun de ses pieds pour grimper sur un poteau, a délogé éperon. Non sans écueil... Et maints sourires ironiques. Et bien entendu, la recherche devra conduire vers les termes français techniques évincés par des calques de l'anglais comme pylône en remplacement de tour (tower).

\section{DISCUSSIONS EN COMITÉ}

Les terminologues soumettent en moyenne une vingtaine de définitions à chaque réunion du comité. Ces rencontres ont lieu à intervalles fixes, habituellement aux trois semaines. Le calendrier de travail est donc établi à partir du nombre de termes contenus dans la nomenclature.

Quelques jours avant la réunion, les terminologues font parvenir la liste des définitions établie aux membres du comité. Ils y joignent les définitions modifiées des termes étudiés à la réunion précédente et corrigées en fonction des décisions prises. Les spécialistes sont donc en mesure de prendre connaissance des définitions et de consulter leurs collègues.

En réunion, on fait la lecture des définitions. Les membres du comité apportent leurs commentaires. Les discussions s'engagent, parfois longues, enflammées à l'occasion, tantôt vives, tantôt cordiales; elles peuvent aussi donner lieu à des débats volcaniques, ou même ne pas susciter la moindre opposition. Comme des alchimistes à la recherche du secret de l'or, les terminologues doivent découvrir l'art du consensus. Patience et persévérance sont recommandées! Dans certains cas, les spécialistes iront même jusqu'à dessiner au tableau une pièce d'équipement afin de débroussailler les notions. Ou apportent à la réunion l'objet du litige: bout de câble, isolateur, entretoise, règlement, article de loi, etc. Les terminologues ou le coordonnateur note(nt) les remarques, les suggestions et les décisions qui se prennent.

Des visites d'installations s'inscrivent au programme: un centre de formation pour monteurs et jointeurs, un poste où nous observons une remontée de câbles, ou bien une usine de fabrication de câbles souterrains. Ces visites sont précieuses. D'abord, elles permettent de voir le matériel, de comprendre son utilisation. Elles servent aussi à prendre contact avec les usagers de la terminologie, à s'en faire des alliés, voire des collaborateurs.

Dans un vocabulaire, les illustrations jouent un rôle complémentaire car elles facilitent la compréhension de la définition, tout particulièrement lorsqu'il s'agit de dispositifs complexes ou d'éléments qui s'intègrent dans un ensemble. (Voir l'illustration du câble isolé en annexe.) La validation des illustrations est essentielle et se fait toujours en comité. L'idéal serait qu'elle progresse au même rythme que les travaux, mais il y a 
loin de la coupe aux lèvres... Les terminologues aidés de spécialistes doivent souvent consacrer plusieurs heures à la recherche et au choix des illustrations en bout de piste.

De définitions en définitions s'élabore le corpus qui varie de 125 à plus de 1000 entrées. Lorsque tous les termes ont été définis, scrutés à la loupe et finalement validés par le comité, les terminologues préparent l'édition provisoire qui comprend tant les définitions que les illustrations. Viennent ensuite l'avant-propos, les notes liminaires, la bibliographie, la table des matières et l'index s'il y a lieu. Cette édition est envoyée en consultation dans les unités administratives touchées directement ou indirectement par le vocabulaire, de même qu'aux professionnels de l'écrit. Les terminologues compilent les commentaires reçus: suggestions d'ajouts, de suppressions ou de modifications. Le comité se penche sur cette moisson et décide de sa pertinence. La consultation élargie permet d'enrichir le vocabulaire et de l'ajuster plus étroitement aux besoins des utilisateurs. Après l'intégration des derniers commentaires, les terminologues préparent le manuscrit.

\section{ÉDITION}

L'édition comprend la correction d'épreuves, le graphisme, la typographie, le montage, l'impression et la diffusion. C'est la partie confiée aux responsables du graphisme, à l'exception de la correction d'épreuves et de la diffusion. Les membres du comité devront encore se prononcer quant au choix de la maquette de la couverture.

Dernière étape et non la moindre, la diffusion contribue au succès de l'implantation de la terminologie dans l'entreprise. Pour que cette opération soit efficace, il convient de bien identifier le public cible. Les membres du comité, les professionnels de l'écrit et de la communication, tous sont mis à contribution pour promouvoir le nouveau produit.

Après des envois massifs à des groupes déterminés, une publicité dans le journal de l'entreprise assurera un bon départ au nouveau-né : article spécial ou chronique faisant la synthèse des travaux du comité. La publication sera transmise à l'Office de la langue française qui diffusera cette nouvelle terminologie par le biais de sa banque et sera également envoyée à certains services de terminologie d'autres entreprises. Un lancement soulignera souvent l'événement.

\section{ET PUIS APRÈS...}

Et puis après, l'aventure recommence! Des besoins nouveaux surgissent et un autre projet s'organise. Depuis les années 80 , nous avons sans cesse raffiné nos méthodes de recherche thématique et constitué une famille de vocabulaires et de lexiques dont nous sommes plutôt fiers, ma foi!

- Vocabulaire illustré des lignes aériennes de transport et de distribution d'électricité en quatre fascicules plus un index

- Vocabulaire illustré des lignes souterraines de transport et de distribution

d'électricité en deux fascicules

- Vocabulaire de la turbine Francis

- Vocabulaire des interconnexions (à paraître)

- Lexique de l'exploitation automatisée du réseau en deux fascicules (deuxième fascicule à paraître)

- Lexique des régulateurs de vitesse

- Vocabulaire de la fonction commerciale

- Vocabulaire des termes d' architecture et du bâtiment

- Vocabulaire du contrôle de gestion

- Terminologie de la demande en deux fascicules. 


\section{PIERSONNES-RESSOURCES}

Nicole April, service Édition et Communication écrite, Hydro-Québec Jean-Marc Lambert, service Terminologie et Diffusion, Hydro-Québec

\section{6. câble (isolé)}

Ensemble constitué par un ou plusieurs conducteurs isolés et mécaniquement solidaires, généralement sous un ou des revêtements protecteurs (gaine externe, armure, revêtement d'assemblage, etc.) II peut comporter en plus un ou plusieurs conducteurs non isolés. $(1,5,11,13)$

Insulated cable

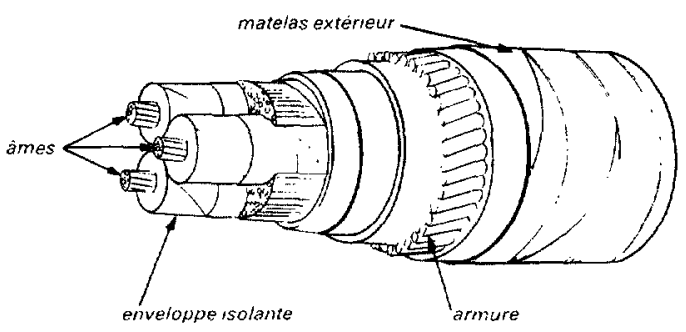

câble tripolatre

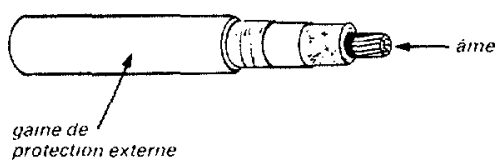

cable umipolatre

\section{BIBLIOGRAPHIE}

AUGER, Pierre et Louis-Jean ROUSSEAU (1978): Méthodologie de la recherche terminologique, Études, recherches et documentation, Québec, Éditeur officiel du Québec.

DUBUC, Robert (1985): Manuel pratique de terminologie, $2^{\mathrm{e}}$ éd., revue et augmentée, Montréal, Linguatech.

VIDAL, Gigi (1989): «La terminologie thématique à Hydro-Québec», Exposé présenté au Congrès annuel de la Société des traducteurs du Québec, Atelier «L'état actuel de la terminologie», Montréal, 13 juin. 